\title{
BOUNDEDNESS FOR SOME RATIONALLY CONNECTED THREEFOLDS IN $\mathbb{P}^{6}$
}

\author{
MARIAN APRODU AND MATEI TOMA
}

\begin{abstract}
We prove boundedness of rationally-connected threefolds in $\mathbb{P}^{6}$ under some extra-assumptions.
\end{abstract}

\section{INTRODUCTION}

Hartshorne and Lichtenbaum conjectured that rational surfaces in $\mathbb{P}^{4}$ form a bounded family. This famous conjecture was proved in a more general setup by Ellingsrud and Peskine EP89. Since then, the higher-dimensional case has become the center of attention. Precisely, it is expected that there are finitely many components of the Hilbert scheme parameterizing smooth subvarieties $X$ not of general type in $\mathbb{P}^{N}$ of dimension $\operatorname{dim}(X) \geq N / 2$ (since any variety $X$ can be embedded in $\mathbb{P}^{2 \operatorname{dim}(X)+1}$ the problem of boundedness only makes sense in this range). M. Schneider [Sch92] has given an affirmative answer, for $\operatorname{dim}(X) \geq$ $(N+2) / 2$. Hence the remaining question is whether or not the same is true for $N \in\{2 \operatorname{dim}(X)-1,2 \operatorname{dim}(X)\}$. The case of threefolds in $\mathbb{P}^{5}$ has been settled in BOSS, which brings us to the next non-trivial case of smooth threefolds $X$ in $\mathbb{P}^{6}$, the object of this note. Recall that only finitely many components of the Hilbert scheme of smooth varieties can appear if the degree is bounded.

We prove:

Theorem 1.1. Let $X$ be a smooth rationally connected threefold in $\mathbb{P}^{6}$ not contained in any fourfold of degree $\leq 34$ and denote by $S$ a general hyperplane section. If $K_{S}^{2} \leq 9$, then the degree of $X$ is bounded by $34^{3}$.

The condition $K_{S}^{2} \leq 9$ is realised if either $X$ is covered by lines, or $\left|K_{X}+H\right|=\emptyset$, where $H$ denotes the hyperplane class, or $S$ is not of general type, Corollary 5.1 The hypothesis on rational connectedness will be used only through its consequences on cohomology vanishing, i.e. $h^{i}\left(\mathcal{O}_{X}\right)=0$ for $i \geq 1$.

Some other boundedness results in the same spirit have been obtained by Sabatino Sa05. Precisely, in loc. cit. it is assumed that the general hyperplane section of $X$ is a ruled surface.

It follows directly from IT94 that subcanonical varieties not of general type form a bounded family. Hence we are mainly concerned with the case of non-subcanonical subvarieties, although this hypothesis will not be used.

The main technical ingredients used in the proof are the following: the lifting theorem of Chiantini-Ciliberto CC93, the bounds on the genus of curves in $\mathbb{P}^{4}$ of

\footnotetext{
Marian Aprodu thanks IECN Nancy and IHES Bures-sur-Yvette for hospitality during the preparation of this work. Marian Aprodu was partly supported by LEA MathMode, and by the CNCS-UEFISCDI grant PN-II-ID-PCE- 2011-3-0288, contract no. 132/05.10.2011. Matei Toma was partly supported by LEA MathMode.
} 
Chiantini-Ciliberto-di Gennaro CCdG95, the semi-positivity of Schur polynomials of Fulton-Lazarsfeld [FL93, and the Hodge index formula for some divisors in $X$.

\section{Notation, Setup}

The general setup is the following.

$X \subset \mathbb{P}^{6}$ is a rationally connected smooth variety of dimension 3 ,

$H:=\mathcal{O}_{X}(1)$ the hyperplane section bundle on $X$,

$K:=K_{X}$ the canonical bundle of $X$,

$h:=c_{1}(H) \in H^{2}(X, \mathbb{Z})$ the class of $H$,

$k:=c_{1}(K) \in H^{2}(X, \mathbb{Z})$ the class of $K$,

$N:=\left.N\right|_{X \mid \mathbb{P}^{6}}$ the normal bundle of $X$,

$S \in|H|$ a general hyperplane section,

$H_{S}:=\left.H\right|_{S}$ the induced very ample bundle on $S$,

$C \in\left|H_{S}\right|$ a general sectional curve,

$H_{C}:=\left.H\right|_{C}$ the induced very ample bundle on $C$,

$d:=\left(H^{3}\right)$ the degree of $X$,

$g:=g(C)$ the sectional genus,

$\delta:=2 g-2=h^{2} \cdot k+2 h^{3}$ the degree of $K_{C}$,

$c_{i}:=c_{i}(X) \in H^{2 i}(X, \mathbb{Z}), i \in\{1,2,3\}$ the Chern classes of $X$,

$n_{i}:=c_{i}(N) \in H^{2 i}(X, \mathbb{Z}), i \in\{1,2,3\}$ the Chern classes of the normal bundle,

$\chi:=\chi\left(\mathcal{O}_{S}\right)$ the Euler characteristic of $S$,

$u:=h^{1,1}(S)$ the Picard number of $S$,

$v:=c_{1}(N(-H))^{3}$.

Note that $h^{i}\left(\mathcal{O}_{X}\right)=0$ for all $i \geq 1$ Ko96, Corollary IV.3.8.

\section{Identities}

In this section we record a number of useful relations in connection with our setup.

Lemma 3.1. $S$ is a regular surface i.e. $q(S)=0$. Moreover $\chi=\chi(K+H)+1=$ $h^{0}(K+H)+1$ and hence $p_{g}(S)=0$ if and only if $\left|K_{X}+H\right|=\emptyset$.

Proof. Apply $h^{2}\left(\mathcal{O}_{X}(-1)\right)=0$ (from Kodaira vanishing) and the vanishing of $h^{1}\left(\mathcal{O}_{X}\right)$ and $h^{2}\left(\mathcal{O}_{X}\right)$ to the exact sequence

$$
0 \rightarrow \mathcal{O}_{X}(-1) \rightarrow \mathcal{O}_{X} \rightarrow \mathcal{O}_{S} \rightarrow 0
$$

Lemma 3.2. $c_{2}(S)=2 \chi+u$ and $K_{S}^{2}=10 \chi-u$.

Proof. From the Gauss-Bonnet formula, we have $c_{2}(S)=\chi_{\text {top }}(S)=2-2 b_{1}(S)+$ $b_{2}(S)$, from where, applying Lemma 3.1, we obtain $c_{2}(S)=2+b_{2}(S)=2 \chi+u$.

For the second relation, apply Noether's formula on $S$.

$$
\chi=\frac{1}{12}\left(K_{S}^{2}+\chi_{\text {top }}(S)\right),
$$

which implies $K_{S}^{2}=12 \chi-(2 \chi+u)$.

Lemma 3.3. $k \cdot c_{2}=-24$. 
Proof. From the Hirzebruch-Riemann-Roch formula, we have $\chi\left(\mathcal{O}_{X}\right)=c_{1} \cdot c_{2} / 24$, and from rational-connectedness we have $\chi\left(\mathcal{O}_{X}\right)=1$.

Lemma 3.4. The Chern classes of $N$ are the following:

(1) $n_{1}=7 h+k$;

(2) $n_{2}=21 h^{2}+7 h \cdot k+k^{2}-c_{2}$;

(3) $n_{3}=35 h^{3}+21 h^{2} \cdot k+7 h \cdot k^{2}+k^{3}-7 h \cdot c_{2}-c_{3}+48$.

Proof. We use the normal sequence

$$
\left.0 \rightarrow T_{X} \rightarrow T_{\mathbb{P}^{6}}\right|_{X} \rightarrow N \rightarrow 0
$$

which implies that $c(N)=c\left(\left.T_{\mathbb{P}^{6}}\right|_{X}\right) / c(X)$. Obviously

$$
\frac{1}{c(X)}=1-c_{1} t+\left(c_{1}^{2}-c_{2}\right) t^{2}+\left(-c_{1}^{3}+2 c_{1} c_{2}-c_{3}\right) t^{3}
$$

i.e.

$$
\frac{1}{c(X)}=1+k t+\left(k^{2}-c_{2}\right) t^{2}+\left(k^{3}+48-c_{3}\right) t^{3}
$$

On the other hand, since $c\left(T_{\mathbb{P}^{6}}\right)=(1+h t)^{7}$, we obtain

$$
c_{1}\left(\left.T_{\mathbb{P}^{6}}\right|_{X}\right)=7 h, c_{2}\left(\left.T_{\mathbb{P}^{6}}\right|_{X}\right)=\left(\begin{array}{l}
7 \\
2
\end{array}\right) h^{2}=21 h^{2}, c_{3}\left(\left.T_{\mathbb{P}^{6}}\right|_{X}\right)=\left(\begin{array}{l}
7 \\
3
\end{array}\right) h^{3}=35 h^{3} .
$$

This implies

$$
c(N)=\left(1+7 h t+21 h^{2} t^{2}+35 h^{3} t^{3}\right) \cdot\left(1+k t+\left(k^{2}-c_{2}\right) t^{2}+\left(k^{3}+48-c_{3}\right) t^{3}\right) .
$$

Lemma 3.6. $n_{3}=d^{2}$.

Proof. This is the double-point formula [LMS75.

Lemma 3.7. We have the following numerical relations:

(1) $h^{2} \cdot k=-2 d+\delta$;

(2) $h \cdot k^{2}=3 d-2 \delta+10 \chi-u$;

(3) $k^{3}=-4 d-24 \delta-120 \chi+12 u+v$;

(4) $h \cdot c_{2}=d-\delta+2 \chi+u$.

(5) $c_{3}=3 d-10 \delta-64 \chi-2 u+v-d^{2}+48$;

Proof. For (1), by adjunction we have $\delta=(k+2 h) \cdot h^{2}$, and hence $h^{2} \cdot k=-2 d+\delta$.

For (2) we apply Lemma 3.2 $K_{S}^{2}=10 \chi-u$. On the other hand

$$
K_{S}^{2}=(k+h)^{2} \cdot h=h^{3}+2 h^{2} \cdot k+h \cdot k^{2}
$$

implying $h \cdot k^{2}=10 \chi-u-d-2(-2 d+\delta)$.

For (3) we use the definition of $v$. Note that $c_{1}(N(-1))=4 h+k$, from Lemma 3.4, and hence

$$
v=64 h^{3}+48 h^{2} \cdot k+12 h \cdot k^{2}+k^{3} .
$$

The preceding formulae show that

$$
k^{3}=v-64 d-48(-2 d+\delta)-12(3 d-2 \delta+10 \chi-u) .
$$

For (4) remark that $h \cdot c_{2}=c_{2}\left(\left.T_{X}\right|_{S}\right)$. The exact sequence

$$
\left.0 \rightarrow T_{S} \rightarrow T_{X}\right|_{S} \rightarrow N_{S \mid X} \rightarrow 0
$$


implies that (note that $\left.N_{S \mid X}=H_{S}\right) h \cdot c_{2}=c_{2}(S)+c_{1}(S) \cdot H_{S}$. From Lemma 3.2 it follows that $h \cdot c_{2}=2 \chi+u-(k+h) \cdot h^{2}$.

For (5), apply Lemma 3.4 Lemma 3.6 and the relations above: $c_{3}=35 h^{3}+$ $21 h^{2} \cdot k+7 h \cdot k^{2}+k^{3}-7 h \cdot c_{2}-n_{3}+48=35 d+21(-2 d+\delta)+7(3 d-2 \delta+10 \chi-$ $u)+(-4 d-24 \delta-120 \chi+12 u+v)-7(d-\delta+2 \chi+u)-d^{2}+48$.

\section{INEQUALITIES}

This section is devoted to some useful inequalities between the given invariants.

4.1. A Lifting Theorem. CC93 It is known Ro37 that if the sectional curve $C$ is contained in a hypersurface of degree $s$ in $\mathbb{P}^{4}$, with $s^{2}<d$, then both $S$ and $X$ are contained in hypersurfaces of degree $s$ in $\mathbb{P}^{5}$, and $\mathbb{P}^{6}$ respectively.

We shall need a version of this result, which is a special case of Theorem 0.2 CC93.

Theorem 4.1. If the general sectional curve $C$ of $X$ is contained in a surface in $\mathbb{P}^{4}$ of degree $s$ such that $d>\frac{(s-1)(s-3)}{2}+8 s-3$ then $X$ is contained in a 4 -fold of degree $s$ in $\mathbb{P}^{6}$.

This result will be used together with the bounds of the genus of $C$, see below.

4.2. Bounds on the genus of $C$. We shall apply the main result of [CCdG95] in our setup; the general sectional curve $C$ is non-degenerate.

Theorem 4.2. Notation as above. Assume that $C$ is not contained in a surface of even degree $s \geq 11$ with $d>s^{3}$. Then the genus of $C$ is bounded by

$$
\frac{d^{2}}{2 s}+\frac{d}{2}\left(\frac{s}{2}-3\right)+\frac{3 s^{2}-20}{8} \text {. }
$$

When making the substitutions in the main result of [CCdG95] we do not take care of optimality of the bound. A similar bound can be found for odd $s$, either by applying directly loc. cit. or replacing $s$ by $s-1$ in the statement above.

4.3. Schur polynomials. The bundle $N(-1)$ is globally generated, hence by [FL93, the associated Schur polynomials

$$
\begin{gathered}
s_{(1)}:=c_{1}(N(-1)), s_{(20)}:=c_{2}(N(-1)), s_{(300)}:=c_{3}(N(-1)), \\
s_{(11)}:=\left(c_{1}^{2}-c_{2}\right)(N(-1)), s_{(210)}:=\left(c_{1} \cdot c_{2}-c_{3}\right)(N(-1)) \\
s_{(111)}:=\left(c_{1}^{3}-2 c_{1} \cdot c_{2}+c_{3}\right)(N(-1))
\end{gathered}
$$

are semi-positive, in particular $s_{(1)} \cdot h^{2}, s_{(20)} \cdot h$ and $s_{(11)} \cdot h$ are non-negative. We compute all these non-negative numbers.

Lemma 4.3. Notation as above. We have

(1) $s_{(1)} \cdot h^{2}=2 d+\delta$;

(2) $s_{(20)} \cdot h=2 d+4 \delta+8 \chi-2 u$;

(3) $s_{(11)} \cdot h=d+2 \delta+2 \chi+u$;

(4) $s_{(300)}=-5 d-5 \delta-8 \chi+2 u+d^{2}$;

(5) $s_{(210)}=4 d-3 \delta-30 \chi-3 u+v+24-d^{2}$;

(6) $s_{(111)}=-3 d+11 \delta+68 \chi+4 u-v-48+d^{2}$.

Proof. We easily show that $c_{1}(N(-1))=n_{1}-3 h, c_{2}(N(-1))=n_{2}+3 h^{2}-2 h \cdot n_{1}$, $c_{3}(N(-1))=n_{3}-h^{3}+h^{2} \cdot n_{1}-h \cdot n_{2}$. We apply next Lemma 3.4, 3.3, 3.6, 3.7. 


\subsection{Hodge-index Theorem.}

Proposition 4.4. Let $D$ be a smooth divisor on $X$. Then

$$
(H \cdot K \cdot D)^{2} \geq\left(H^{2} \cdot D\right)\left(K^{2} \cdot D\right) \text {. }
$$

Proof. Write $(H \cdot K \cdot D)=\left(\left.\left.H\right|_{D} \cdot K\right|_{D}\right),\left(H^{2} \cdot D\right)=\left(\left.H\right|_{D} ^{2}\right),\left(K^{2} \cdot D\right)=\left(\left.K\right|_{D} ^{2}\right)$ and apply the usual Hodge-index theorem on $D$ to $\left.H\right|_{D}$ and $\left.K\right|_{D}$.

Remark 4.5. Applying Hodge theorem for a linear combination $a D_{1}+b D_{2}$ of divisors does not give anything new apart from the inequalities obtained for $D_{1}$ and $D_{2}$.

We can apply Proposition 4.4 to two different divisors on $X$. One is $H$ itself and the other one is $4 H+K$; recall that $\operatorname{det}(N(-1))=4 H+K$ is globally generated.

Corollary 4.6. $(3 d+6 \delta+10 \chi-u)^{2} \geq v(2 d+\delta)$ and $\delta^{2}-(2 \delta+10 \chi-u) d+d^{2} \geq 0$.

\section{Boundedness of ThE DEGREe}

\section{Proof of Theorem 1.1.}

Suppose that $d>34^{3}$. From the hypothesis, we know that $10 \chi-u \leq 9$. Note that

$$
s_{(20)} \cdot h+s_{(11)} \cdot h=3 d+6 \delta+10 \chi-u \geq 0 .
$$

From corollary 4.6, we obtain

$$
v \leq \frac{(3 d+6 \delta+10 \chi-u)^{2}}{2 d+\delta} \leq \frac{(3 d+6 \delta+9)^{2}}{2 d+\delta}
$$

On the other hand, from the non-negativity of $s_{(210)}$, we have

$$
v \geq d^{2}-4 d+3 \delta+30 \chi+3 u-24 \geq d^{2}-4 d+3 \delta+9
$$

whence

$$
33 \delta^{2}+\left(-d^{2}+34 d+99\right) \delta+\left(81+17 d^{2}+36 d-2 d^{3}\right) \geq 0
$$

Since for $d>34^{3}$, the expression $81+17 d^{2}+36 d-2 d^{3}$ is clearly negative, we have

$$
\delta \geq \frac{d^{2}-34 d}{33}-3
$$

From theorem 4.1 and theorem 4.2 applied for $s=34$, it follows that

$$
\delta=2 g-2 \leq \frac{d^{2}}{s}+d\left(\frac{s}{2}-3\right)+\frac{3 s^{2}}{4}-7=\frac{d^{2}}{34}+14 d+860 .
$$

These two opposite inequalities for $\delta$ yield

$$
\frac{d^{2}}{34}+14 d+860 \geq \frac{d^{2}-34 d}{33}-3
$$

which contradicts the assumption $d>34^{3}$.

Corollary 5.1. Let $X$ be a smooth rationally connected threefold in $\mathbb{P}^{6}$ not contained in any fourfold of degree $\leq 34$. Then the degree of $X$ is bounded by $34^{3}$ if one of the following conditions is satisfied:

(1) $X$ is covered by lines;

(2) the general hyperplane section $S$ of $X$ is not of general type;

(3) $\left|K_{X}+H\right|=\emptyset$, where $H$ denotes the hyperplane class. 
Proof. We prove that in each of the three cases, we have $K_{S}^{2} \leq 9$.

If $S$ is not of general type, then the conclusion follows from the classification of surfaces (note that $K^{2}$ decreases when we blow up).

If $X$ is covered by lines, then $\left|K_{X}+H\right|$ is empty. Indeed, by [R12], it follows that for $\ell$ a general line in a covering family, the restriction $\left.\Omega_{X}^{1}\right|_{\ell} \cong \mathcal{O}(-2) \oplus \mathcal{O}(a) \oplus \mathcal{O}(b)$ with $-1 \leq a, b \leq 0$ (i.e. lines are free, standard). Hence $K_{X} \cdot \ell \leq-2$ and, since $H \cdot \ell=1,\left(K_{X}+H\right) \cdot \ell \leq 1$. Hence $K_{X}+H$ cannot be effective.

Under the assumption $\left|K_{X}+H\right|=\emptyset$, from Lemma 3.1, we infer that $p_{g}(S)=0$, and hence $\chi=1$, which implies that $10 \chi-u \leq 9$.

\section{REFERENCES}

[BOSS] R. Braun, G. Ottaviani, M. Schneider, F.-O. Schreyer, Boundedness for non-general type 3-folds in $\mathbb{P}_{5}$, Complex Analysis and Geometry (1993) 311-338.

[CC93] L. Chiantini, C. Ciliberto, A few remarks on te lifting problem, Journées de géométrie algébrique d'Orsay, Astérisque 218 (1993) 95-109.

[CCdG95] L. Chiantini, C. Ciliberto, V. Di Gennaro, The genus of curves in $\mathbb{P}^{4}$ verifying certain flag conditions, Manuscripta Math. 89 (1995) 119-134.

[EP89] G. Ellingsrud, Ch. Peskine, Sur les surfaces lisses de $\mathbb{P}_{4}$, Invent. Math. 95 (1989) 1-11.

[FL93] W. Fulton, R. Lazarsfeld, Positive polynomials for ample vector bundles, Ann. of Math. 118 (1983) 35-60.

[IT94] P. Ionescu, M. Toma, Boundedness for some special families of embedded manifolds, Contemp. Math. 162 (1994) 215-225.

[IR12] P. Ionescu, F. Russo, Manifolds covered by lines and the Hartshorne conjecture for quadratic manifolds, American J. Math., to appear.

[Ko96] J. Kollár, Rational curves on algebraic varieties, Springer Verlag, 1996.

[LMS75] A. T. Lascu, D. Mumford, D. B. Scott, The self-intersection formula and the formuleclef, Math. Proc. Camb. Phil. Soc. 78 (1975), 117-123.

[Sa05] P. Sabatino, Boundedness for threefolds in $\mathbb{P}^{6}$ containing a smooth ruled surface as hyperplane section, Rev. Mat. Complut. 18 (2005) 363-375.

[Ro37] L. Roth, On the projective classification of surfaces, Proc. London Math. Soc 42 (1937) 142-170.

[Sch92] M. Schneider, Boundedness of low-codimensional submanifolds of projective space, Int. J. Math. 3 (1992) 397-399.

Marian Aprodu: Romanian Academy, Institute of Mathematics "Simion Stoilow" P.O. Box 1-764, RO 014700, Bucharest, Romania

E-mail address: marian.aprodu@imar.ro

Matei Toma: Institut Elie Cartan, UMR 7502, Université de Lorraine, CNRS, INRIA, Boulevard des Aiguillettes, B.P. 70239, 54506 Vandoeuvre-lès-Nancy Cedex, France

E-mail address: Matei.Toma@univ-lorraine.fr 